\title{
Light concentrators for large-volume detector at the Baksan Neutrino Observatory
}

\author{
A. N. Fazliakhmetov, ${ }^{1,2, *}$ V. N. Gavrin, ${ }^{1}$ T. V. Ibragimova, ${ }^{1}$ B. K. Lubsandorzhiev, ${ }^{1}$ \\ A. D. Lukanov , ${ }^{1}$ Yu. M. Malyshkin, ${ }^{1,3}$ A. Yu. Sidorenkov, ${ }^{1}$ A. A. Shikhin, ${ }^{1}$ \\ O. Yu. Smirnov, ${ }^{3}$ N. A. Ushakov, ${ }^{1}$ E. P. Veretenkin ${ }^{1}$ and D. M. Voronin ${ }^{1}$ \\ ${ }^{1}$ Institute for Nuclear Research of Russian Academy of Sciences, Moscow 117312, Russia \\ ${ }^{2}$ Moscow Institute of Physics and Technology (National Research University), Dolgoprudny 117303, Russia \\ ${ }^{3}$ Joint Institute for Nuclear Research, Joliot Curie 6, Dubna, Moscow Region 141980, Russia \\ E-mail: fazliakhmetov@phystech.edu
}

At the Baksan Neutrino Observatory deployed in the North Caucasus mountains, it is proposed to develop, at a depth corresponding to about 4700 mwe, a large-volume neutrino detector based on a liquid scintillator with a target mass of $10 \mathrm{kt}$. The main physics goals of the detector are low-energy neutrino physics, astrophysics and geophysics. The highest possible light yield is crucial for such detectors. To improve light yield and energy resolution in large-volume neutrino detectors, light concentrators are often mounted on photomultiplier tubes to increase the detection efficiency of optical photons from scintillation or Cherenkov light induced by charged particles. We present the results of recent R\&D work aimed to develop light concentrators for the Baksan large-volume liquid scintillator neutrino detector.

$37^{\text {th }}$ International Cosmic Ray Conference (ICRC 2021)

July 12 th - 23rd, 2021

Online - Berlin, Germany

\footnotetext{
*Presenter
} 


\section{Introduction}

A new large-volume Baksan neutrino telescope (10 kt liquid scintillator) will be developed at the Baksan Neutrino Observatory of the Institute for Nuclear Research Russian Academy of Sciences at a depth of about 4700 mwe (meter water equivalent) [1],[2], [3]. The main physics goals of the detector are low-energy neutrino physics, geophysics and astrophysics. The highest possible light yield is crucial for detector development. Mounting light concentrators to photomultiplier tubes (PMTs) is one of the ways to increase light collection efficiency. Such device takes light incident on an entrance aperture over a given angular range and reflects by a curved surface to a smaller exit aperture of the PMT photocathode. Additionally, concentrators can reduce total experiment costs by minimizing the required number of PMTs. Light concentrators based on Winston cone [4] was successfully used by several neutrino experiments, for example, the SNO [5] and Borexino [6] and imaging atmospheric Cherenkov telescopes. We present the results of recent R\&D work aimed to develop light concentrators for the Baksan large-volume liquid scintillator neutrino detector.

Section 2 presents the detector prototypes design. The method for optimization of the concentrator shape will be described in Section 3. In Section 4, we present the results of concentrators $\mathrm{R} \& \mathrm{D}$ work.

\section{5t prototype design}

The neutrino telescope project is divided into four stages with increasing detection mass of liquid scintillator target: from $0.5 \mathrm{t}$ prototype to $5 \mathrm{t}$ (second prototype), $100 \mathrm{t}$ (third stage) and the full-scale detector (10kt) on the last phase. The first and second prototypes have a two-zone design. Currently, the first stage of the project is being completed, the second prototype (5t) is under construction.

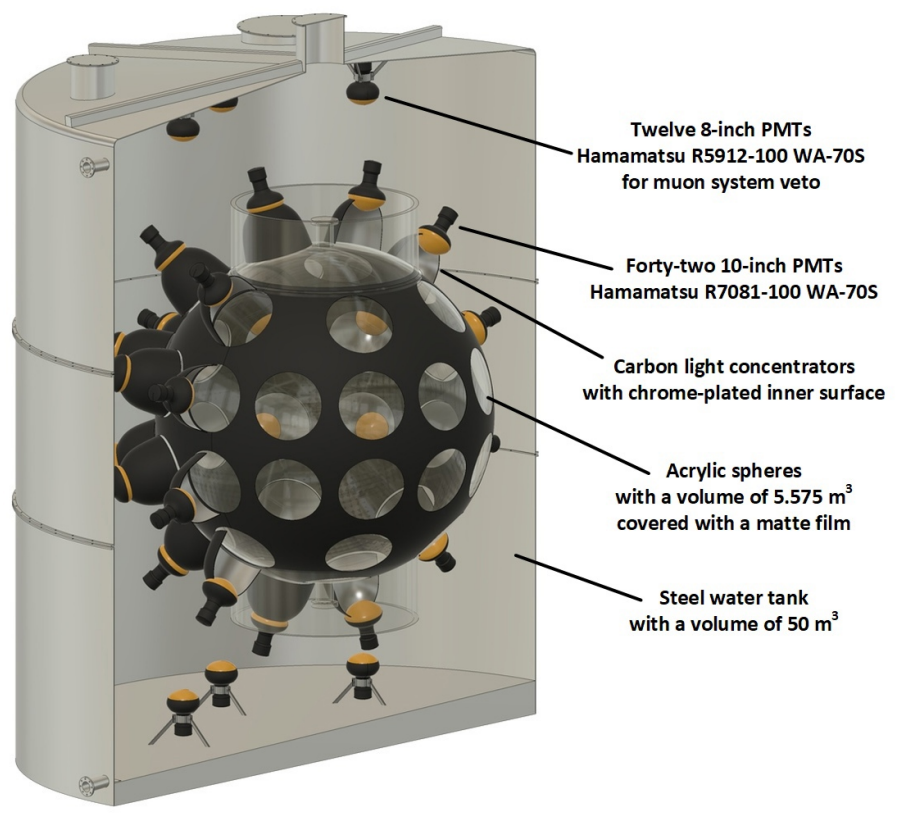

Figure 1: 5t detector prototype design 
Figure 1 shows $5 t$ detector prototype design. The central zone is an acrylic sphere filled with an ultrapure liquid scintillator (linear alkylbenzene (LAB) + PPO) and surrounded by a second zone. The second zone is filled with water to protect against external radioactivity and also serves as muon veto. The scintillator sphere is surrounded by 42 10-inch Hamamatsu R7081-100 PMTs (220 mm. diameter photocathode). On the second prototype, we decided to use light concentrators to increase total light yield. Their shape optimisation method is described in the next section.

\section{Concentrator shape optimisation}

The concentrator shape was developed using the String method (also known as Winston cone method [4]) which considers the shape of the photocathode. Figure 2 illustrates the concentrator shape calculation based on based on the String method.

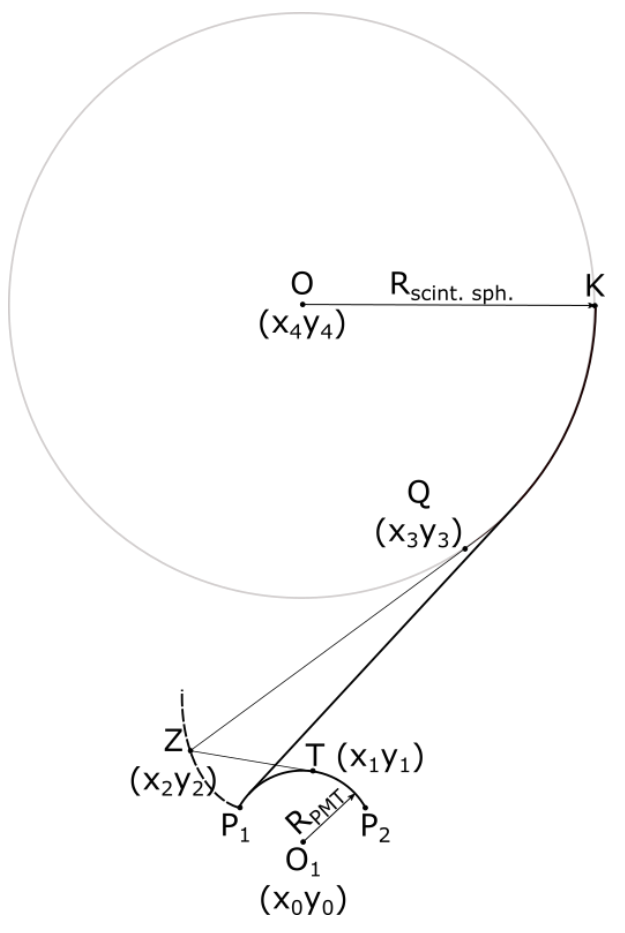

Figure 2: The String method principle illustration.

Between scintillation sphere with the centre at point $O$ and PMT photocathode with curvature centre $O_{1}$, let's stretch the "string". One end of the "string" is tied on point $\mathrm{K}$, another end on point $P_{2}$ which is the right boundary of the photocathode surface. We choose the length of the "string" so that, when taut, it wraps around the photocathode and reaches point $P_{1}$ (the left border of the photocathode). Put the tip of a pencil at point $P_{1}$. Holding the length fixed, we trace out the concentrator profile as the tip moves from $P_{1}$ to the top. Rotating the profile about the axis of symmetry gives the concentrator shape. In $2 \mathrm{D}$ case, any incident rays with $\theta<\theta_{\text {cut-of } f}$ will be reflected onto the photocathode. For 3D case, the light collection efficiency will reduce because in the case of skew incident rays the arch of the photocathode will be lower than at the 2D profile.

Implementation of the string method was done by using python3 programming language with NumPy library. Firstly, we calculate the total length of the string $L_{\text {total }}$ connecting the points 
$K, P_{1}, P_{2}$. Then we randomly chose the point $Z$ with the coordinates $\left(x_{2}, y_{2}\right)$. Emiting a tangent to the scintillation sphere and solving the system of equations:

$$
\begin{gathered}
\left(x_{3}-x_{2}\right)^{2}+\left(y_{3}-y_{2}\right)^{2}=Z Q^{2} \\
\left(x_{3}-x_{4}\right)^{2}+\left(y_{3}-y_{4}\right)^{2}=R_{\text {scint.sph }} .
\end{gathered}
$$

we obtain the coordinates of the touch-point Q. The coordinate of point $T\left(x_{1}, y_{1}\right)$ was calculated in the same way. Having the coordinates of points $K, Q, Z, T, P_{2}$, we can calculate the length of the modified string $L^{\prime}$ and the residual $\left(L_{\text {total }}-L^{\prime}\right)$. Varying coordinate $x_{2}$ of point $Z$ we try to minimize residual. Repeating this algorithm, we obtain a set of points characterizing the cone surface.

\section{Results}

The calculated concentrator shape is presented in Figure 3. The diameter of the exit aper-

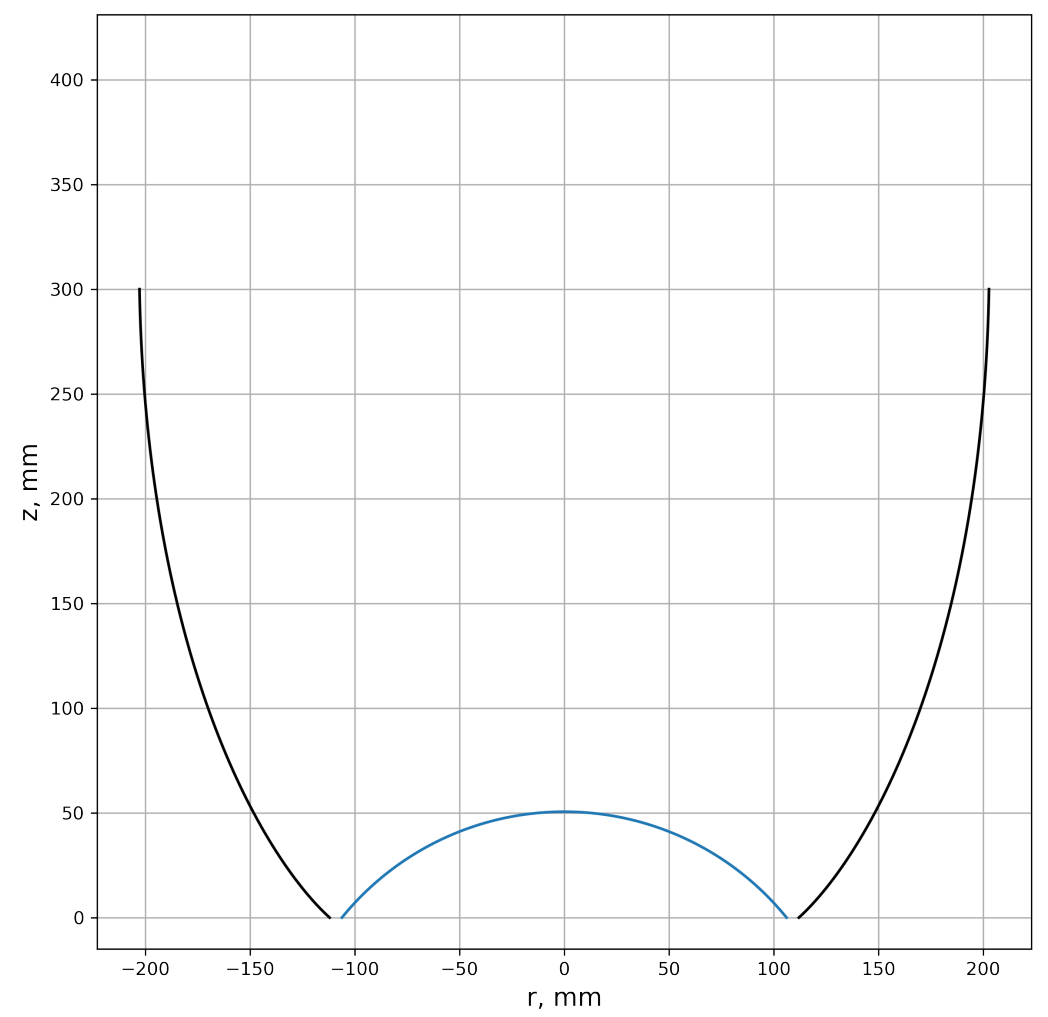

Figure 3: Calculated cone profile.

ture $(224 \mathrm{~mm}$.) is a little bit more than the diameter of the PMT photocathode due to construction reasons. Because of the limited size of the water tank and supporting grid constraints, the total length of the cone is bounded by $300 \mathrm{~mm}$. The cone shape is optimized for this length and given exit aperture size. The entrance aperture diameter is $405 \mathrm{~mm}$. The concentrator increases light yield nearly 3 times compared to bare PMT and $\theta_{\text {cut-of } f} \approx 50^{\circ}$. 
Now the concentrator prototype is under development. It will be made of carbon-fibrereinforced polymer with chrome-based reflective surface inside and matte finish outside. Additionally, both surfaces of the concentrator will be covered with a protective layer.

\section{Acknowledgments}

This work was supported by the Russian Foundation for Basic Research (project No. 20-3290059/20) and the Russian Science Foundation (project No. 17-12-01331).

\section{References}

[1] N.A. Ushakov et al., New large-volume detector at the Baksan Neutrino Observatory: Detector prototype, J. Phys. Conf. Ser. 1787 (2021) 012037.

[2] V.B. Petkov et al., Baksan large volume scintillation telescope: a current status, J. Phys. Conf. Ser. 1468 (2020) 012244.

[3] Y.M. Malyshkin et al., Modeling of a MeV-scale Particle Detector Based on Organic Liquid Scintillator, Nucl. Instrum. Meth. A 951 (2020) 162920 [1909.03229].

[4] R. Winston, J.C. Miñano and P. Benítez, Nonimaging Optics, Academic Press, Burlington (2005), https://doi.org/10.1016/B978-012759751-5/50004-X.

[5] G. Doucas, S. Gil, N. Jelley, L. McGarry, M. Moorhead, N. Tanner et al., Light concentrators for the Sudbury Neutrino Observatory, Nuclear Instruments and Methods in Physics Research Section A: Accelerators, Spectrometers, Detectors and Associated Equipment 370 (1996) 579.

[6] L. Oberauer, C. Grieb, F. Feilitzsch and I. Manno, Light concentrators for Borexino and CTF, Nuclear Instruments and Methods in Physics Research Section A: Accelerators, Spectrometers, Detectors and Associated Equipment 530 (2004) 453. 